doi:10.1515/jrp-2015-0002

\title{
Rotational and gas temperatures of molecular deuterium in a hollow cathode glow discharge
}

\author{
G. Lj. Majstorović ${ }^{1}$ and N. M. Ššović ${ }^{2}$ \\ ${ }^{1}$ University of Defence, Military Academy, 11105 Belgrade, Generala Pavla Jurišića - \\ Šturma 33, Serbia \\ E-mail: gordana.majstorovic@va.mod.gov.rs \\ ${ }^{2}$ University of Belgrade, Faculty of Physics, 11001 Belgrade, P.O. Box 44, Serbia \\ E-mail:nikolas@ff.bg.ac.rs
}

Received: February 17, 2015

\begin{abstract}
We report the results of optical emission spectroscopy measurements of rotational $T_{\text {rot }}$ and translational (gas) temperature of deuterium molecules. The light source was a low-voltage high-pressure hollow cathode (HC) glow discharge with titanium cathode operated in deuterium. The rotational temperature of excited electronic states of $\mathrm{D}_{2}$ was determined from the intensity distribution in the rotational structure of Q-branches of the two Fulcher- $\alpha$ diagonal bands: $\left[\nu^{\prime}=\nu "=2\right]$ and $\left[\nu^{\prime}=\nu "=3\right]$. The population of the excited energy levels, determined from relative line intensities, was used to derive the radial distributions of the temperature of the excited and the ground state of the deuterium molecule.
\end{abstract}

Key words: Optical emission spectroscopy, hydrogen molecule, deuterium molecule, rotational temperature measurement, translational temperature evaluation.

\section{Introduction}

Hydrogen, hydrogen isotopes and hydrogen seeded hollow cathode glow discharges (HCGD) have been extensively used for study of fundamental discharge processes as well as for industrial applications [1]. Area of application of this type of discharge is producing hydrogen by reforming natural gas [2]. Both fundamental and applied research requires an understanding of chemical kinetics and knowledge of the discharge parameters. The translational (gas) temperature $T_{\mathrm{g}}$ of molecules and radicals is a very important plasma parameter, since it determines the rate of chemical reactions. In addition, vibrationally excited deuterium and hydrogen molecules play a significant role in creating negative ion sources or in the chemistry of weakly ionized hydrogen plasmas [3-4]. Unfortunately, the electronic-vibro-rotational (ro-vibrational) transitions of $\mathrm{H}_{2}$ or $\mathrm{D}_{2}$ ground state are in the 
medium infrared region which is inconvenient for application of standard emission and absorption techniques for $T_{\mathrm{g}}$ measurements. To overcome this problem and to measure $T_{\mathrm{g}}$ of such an important molecule, different laser techniques were developed: coherent anti-stokes Raman scattering (CARS) [5-6], laser-induced fluorescence (LIF) [7,8]. Nevertheless, for practical reasons, the search for a simple, reliable optical emission spectroscopic (OES) technique continued, see e.g. Refs. [9-15]. If the OES technique can be applied with standard laboratory equipment in the visible region of the spectrum it would be of importance for monitoring and the control of various hydrogen or hydrogen isotopes plasma assisted technologies.

The Fulcher- $\alpha$ band spectra has been already used for measurement of ro-vibrational population of hydrogen or deuterium molecules in divertor plasmas of fusion devices [16], for plasma simulators [13], laboratory plasma diagnostics [17], including negative ion sources [18-19]. Recently, the rotational, vibrational and translational temperatures of hydrogen molecules in HCGD [20] were determined using relative line intensities belonging to Fulcher- $\alpha$ diagonal band $\left(d^{3} \Pi_{u} \rightarrow a^{3} \Sigma_{g}^{+}\right.$electronic transition; P-, Q- and R-branches; $\left.\nu^{\prime}=\nu^{\prime \prime}=0\right)$. However, the use of line intensities within the Q-branch of the $\left[\nu^{\prime}=\nu^{\prime \prime}=2\right]$ band in $d^{3} \Pi_{u} \rightarrow a^{3} \Sigma_{g}^{+}$electronic transition has been proposed for temperature measurement in hydrogen low pressure gas discharges [21]. The goal of the present work was to check the possibility of using the Q-branches of not only the $\left[\nu^{\prime}=\nu^{\prime \prime}=2\right]$ band, but also the $\left[\nu^{\prime}=\nu^{\prime \prime}=3\right]$ band of Fulcher- $\alpha$ system in spectroscopic diagnostics of deuterium HCGD.

The radial distribution of rotational temperatures within $\mathrm{HC}$ are determined as well.

\section{Experimental}

Hollow titanium cathode with two symmetrically positioned kovar anodes is used as the discharge source. The HC tube was $100 \mathrm{~mm}$ long with $6 \mathrm{~mm}$ internal diameter and $1 \mathrm{~mm}$ wall thickness. The construction details of the $\mathrm{HC}$ discharge source were described elsewhere $[20,22]$.

All HCGD experiments were carried out with deuterium (purity of 99.999\%). The continuous flow of $\mathrm{D}_{2}$ was sustained between 50 and $450 \mathrm{~cm}^{3} / \mathrm{min}$ (at room temperature and atmospheric pressure) in the pressure range 2-10 mbar by means of a needle valve and two-stage mechanical vacuum pump. To prevent the oil vapor back streaming from the vacuum pump, a zeolite trap is placed between the discharge chamber and the vacuum pump. The gas pressure measurements were performed at both sides of the discharge tube. During the discharge the difference of the gas pressure between the gas inlet and outlet from the discharge tube was about $30 \%$. The spectroscopic line intensity recordings did not show measurable difference between axially recorded spectra from the gas inlet or outlet side of the discharge tube. The reported results of gas pressure measurement represent an average between gas inlet and outlet pressure.

To operate the discharge in DC mode, a current stabilized power supply $(0-2 \mathrm{kV}, 0$ $100 \mathrm{~mA}$ ) is used. An air-cooled variable $10 \mathrm{~kW}$ ballast resistor is placed in series with the discharge and the power supply. For all measurements, the cathode was grounded.

During the discharge operation the cathode was air cooled with a fan $(110 \mathrm{~mm}$ dia; AC $220 \mathrm{~V} / 13 \mathrm{~W}$ ), placed $150 \mathrm{~mm}$ from the discharge tube. The outer wall $\mathrm{HC}$ temperature (about $320 \mathrm{~K}$ ) was controlled by a K-type thermocouple. 
The radial distribution spectra recordings were performed with unity magnification in equidistant steps perpendicular to the discharge axis, with an estimated spatial resolution of $0.40 \mathrm{~mm}$. For radial intensity measurements the discharge was run between $\mathrm{HC}$ and the rear anode, located at $20 \mathrm{~mm}$ from the cathode. Here, it should be noted that the difference between the results of test measurements with front and rear anode was within experimental uncertainty. All spectroscopic observations of gas discharge in $\mathrm{HC}$ were performed end-on and, in this way, the Abel inversion procedure required to convert integral radial intensity measurement into radial intensity distribution, see, e.g. [23], was avoided. The light from the discharge was focused with an achromat lens (focal length $75.8 \mathrm{~mm}$ ) onto the entrance slit of $2 \mathrm{~m}$ focal length Ebert type spectrometer with $651 \mathrm{~g} / \mathrm{mm}$ refection grating (the reciprocal dispersion of $0.74 \mathrm{~nm} / \mathrm{mm}$ in first diffraction order). All spectral measurements were performed with an instrumental profile very close to Gaussian form with measured full half-width of $0.018 \mathrm{~nm}$. Signals from the CCD detector $(29.1 \mathrm{~mm}$ length, 3648 channels) are A/D converted, collected and processed by PC.

\section{Basics of hydrogen isotopes molecular temperature measurement}

The basics of translational temperature $T_{\mathrm{g}}$ estimation as well as rotational temperature $T_{\text {rot }}$ measurement technique has been described recently, see [20] and references therein. Here, we shall mention only key differences related to the molecular deuterium.

At the present time only Q-branches of Fulcher- $\alpha$ band emission $\left(d^{3} \Pi_{u}^{-} \rightarrow a^{3} \Sigma_{g}^{+}\right)$is used in plasma diagnostics because of its strong visible emission intensity and few perturbations [24]. Namely, upper level energies and wavelengths in most of hydrogen/deuterium bands are not described by standard formulae because of perturbations from other nearby levels. The choice of the Fulcher- $\alpha$, rather then other molecular system was encouraged by this fact. Due to $\Lambda$-type doubling, $d^{3} \Pi_{u}$ state degenerates into the $d^{3} \Pi_{u}^{+}$and $d^{3} \Pi_{u}^{-}$states. The $d^{3} \Pi_{u}^{-}$state can only have a Q-branch, whereas the $d^{3} \Pi_{u}^{+}$has P- and R-branches, in the spontaneous ro-vibrational emission to $a^{3} \Sigma_{g}^{+}$. Since the degenerated $d^{3} \Pi_{u}^{+}$state interacts strongly with the $e^{3} \Sigma_{g}^{+}$state, the P- and R-branches of Fulcher- $\alpha$ are perturbed and relative transition probabilities for these lines differ from the Hönl-London factors [25-26]. Therefore, we used Q-branches from the $d^{3} \Pi_{u}^{-}$state.

\subsection{Rotational and translational temperature}

The logarithm of the spectral line intensity of a ro-vibrational transition $\left(n^{\prime}, \nu^{\prime}, N^{\prime} \rightarrow\right.$ $n ", \nu$ ",$\left.N^{\prime \prime}\right)$ is a linear function of the upper level energy if the populations of the rotational levels in the excited vibronic state $N\left(n^{\prime}, \nu^{\prime}\right)$ are close to Boltzmann's distribution ( $n$ are the quantum numbers describing an electronic state of the molecule; $\nu$ - vibrational and $N$ rotational quantum number). The term value $\left(E_{n^{\prime}, \nu^{\prime}, N^{\prime}}\right.$ in $\left.\mathrm{cm}^{-1}\right)$ of the upper state is a sum of electronic, vibrational and rotational term values of this state $E_{n^{\prime}, \nu^{\prime}, N^{\prime}}=T_{n^{\prime}}+G_{\nu^{\prime}}+F_{N^{\prime}}$

. By plotting $\ln I_{n^{\prime}, \nu^{\prime}, N^{\prime}}^{n^{\prime}, N^{\prime}, v^{4}} g_{\text {a.s. }} H_{N^{\prime} N^{\prime}}$ against the term values for the upper level in case of Boltzmann population distribution, where $v$ is a wave number, $g_{\text {a.s. }}$ - the statistical weight of the $n^{\prime}, \nu^{\prime}, N^{\prime}$ ro-vibrational level, caused by the nuclear spin and the symmetry with respect to the permutation of the nuclei ('a' or 's'), $H_{N^{\prime} N}$ ' - HL factors [26]), a straight line with the slope $h c / k T_{\text {rot }}\left(n^{\prime}, \nu^{\prime}\right)$ should be obtained. This slope can be used for evaluation of rotational temperature $T_{\text {rot }}\left(n^{\prime}, \nu^{\prime}\right)$ of the excited state. It may also be used to experimentally check the validity of the assumed Boltzmann distribution of rotational 
population: deviations from the straight line are deviations from the assumed distribution. For molecular deuterium $g_{\text {a.s. }}=g_{\text {s. }}=2$ for even and $g_{\text {a.s. }}=g_{\text {a. }}=1$ for odd values of the rotational quantum number, while for molecular hydrogen $g_{\text {a.s. }}=g_{\mathrm{s} .}=1$ for even and $g_{\text {a.s. }}=g_{\text {a. }}=3$ for odd values of the rotational quantum number $\mathrm{N}$ see e.g. [27-29].

In the cases of $\Pi \rightarrow \Sigma$ electronic transition $H_{N^{\prime}} N^{\prime \prime}$ may be written as: $\left(N^{\prime \prime}-1\right)$ for P-branch, $(2 N "+1)$ for Q-branch and $(N "+2)$ for R-branch [26].

Within the framework of the model discussed in $[24,30]$ the logarithm of the scaled ro-vibrational population density should be a linear function of the rotational energy in ground $X^{1} \Sigma_{g}^{+}, \nu=0$ vibronic state:

$$
\ln N_{n^{\prime} \nu^{\prime} N^{\prime}}^{*} \equiv \ln \frac{N_{n^{\prime} \nu^{\prime} N^{\prime}}}{g_{\text {a.s. }}\left(2 N^{\prime}+1\right) \tau_{n^{\prime} \nu^{\prime} N^{\prime}}}=-\frac{h c E_{X 0 N}}{k T_{0}\left(n^{\prime}, \nu^{\prime}\right)}+\text { const. }
$$

where $N_{n^{\prime} \nu^{\prime} N^{\prime}}^{*}$ is the scaled ro-vibrational population density, $\tau_{n^{\prime} \nu^{\prime} N^{\prime}}$ the radiative lifetime of the $n^{\prime} \nu^{\prime} N^{\prime}$ level and $E_{X 0 N}$ the ro-vibrational term value of the ground vibronic state. In this case $T_{0}\left(n^{\prime}, \nu^{\prime}\right)$ is the rotational temperature of the ground vibronic state determined from the rotational population density distribution in an excited $\left(n^{\prime}, \nu^{\prime}\right)$ vibrational state. Further, according to $[24,30]$, the temperature $T_{0}\left(n^{\prime}, \nu^{\prime}\right)$ can be considered as a valid estimation of the ground state ro-vibronic temperatutre, i.e. $\mathrm{D}_{2}$ translational temperature $T_{\mathrm{tr}}$.

Here, it should be emphasized that the model assumes Boltzmann distribution in excited electronic-vibrational states. If one makes an additional assumption, that the rotational energy term $F_{N}=B_{n \nu} N(N+1)$ then the following relation between rotational temperatures in the ground and electronically excited states may be obtained:

$$
\frac{T_{0}\left(n^{\prime} \nu^{\prime}\right)}{B_{X 0}}=\frac{T_{\text {rot }}\left(n^{\prime} \nu^{\prime}\right)}{B_{n^{\prime} \nu^{\prime}}}
$$

where $B_{n^{\prime} \nu^{\prime}}$ and $B_{X 0}$ are rotational constants of the excited states $d^{3} \Pi_{u}$ and the ground $X^{1} \Sigma_{g}^{+}(\nu=0)$ state, see Table 1 .

Table 1. Molecular constants for deuterium ground state and Fulcher- $\alpha$ electronic states [25].

\begin{tabular}{cccccc}
\hline \hline State & $T_{\mathrm{e}}\left(\mathrm{cm}^{-1}\right)$ & $\omega_{\mathrm{e}}\left(\mathrm{cm}^{-1}\right)$ & $\omega_{\mathrm{e}} x_{\mathrm{e}}\left(\mathrm{cm}^{-1}\right)$ & $B_{\mathrm{e}}\left(\mathrm{cm}^{-1}\right)$ & $\alpha_{\mathrm{e}}\left(\mathrm{cm}^{-1}\right)$ \\
\hline$d^{3} \Pi_{u}$ & 112707 & 1678.22 & 32.94 & 15.200 & 0.5520 \\
$a^{3} \Sigma_{u}^{+}$ & 95938 & 1885.84 & 35.96 & 17.109 & 0.606 \\
$X^{1} \Sigma_{g}^{+}$ & 0 & 3118.4 & 64.09 & 30.429 & 1.0492 \\
\hline \hline
\end{tabular}

In the case when the rotational population distribution in the state $n^{\prime}, \nu^{\prime}$ is close to Boltzmann's distribution law with rotational temperature $T_{\text {rot }}\left(n^{\prime}, \nu^{\prime}\right)$ of the excited state, and the characteristic time of rotation-translation relaxation $\tau_{\mathrm{RT}}$ for this state is much shorter than the characteristic time of population and deactivation processes, $T_{\text {rot }}\left(n^{\prime}, \nu^{\prime}\right)$ is equal to the translational temperature, i.e. gas temperature [24]. In the opposite extreme case, when the radiative lifetime is much shorter than $\tau_{\mathrm{RT}}$, the rotational collision mixing of neutral species during lifetime of the state $n^{\prime}, \nu^{\prime}$ is not sufficiently efficient to ensure equilibrium of the rotational distribution with the gas temperature. The degree of relationship between the rotational population density distribution in $n^{\prime}, \nu^{\prime}$ state and 
population of ground electronic state should be proven within the framework of excitationdeactivation balance equations, see e.g. [24,30].

\section{Results and discussion}

In a recent study Fulcher- $\alpha$ diagonal bands $\left(\nu^{\prime}=0,1,2,3\right)$ were used for $T_{\text {rot }}$ measurements in HCGD [20]. Here, we shall point out only several important details relevant for Fulcher- $\alpha$ band gas temperature measurement [20]. First, it is important to notice that the Q-branches of Fulcher- $\alpha$ band system are considered free from perturbations while Pand $\mathrm{R}$ - branches are perturbed and relative transition probabilities for these lines differ from the HL factors [24]. Therefore, in [20] we used the Q-branch $\left[\nu^{\prime}=\nu "=0\right]$ of the Fulcher- $\alpha$ band system as the most reliable for temperature estimation. The arguments are the following: (i) the spectral lines of the P- and R- branches are difficult to study since they originate from perturbed levels of the $d^{3} \Pi_{u}^{+}$state [24] and (ii) $T_{\text {rot }}$ is found to be in good agreement with the temperature value derived using semi empirical approach, see Fig. 4 in [20]. Therefore, we investigate the possibility of using R- or P-branches for temperature measurement first.

Now, our discussion will be focused on the Q-branches $\left[\nu^{\prime}=\nu^{\prime \prime}=2\right]$ and $\left[\nu^{\prime}=\nu^{\prime \prime}=3\right]$ on the $d^{3} \Pi_{u}^{-}, \nu^{\prime}, N^{\prime} \rightarrow a^{3} \Sigma_{g}^{+}, \nu ", N^{\prime \prime}$ deuterium molecular system. The studies of direct $d^{3} \Pi_{u}^{-} \leftarrow X^{1} \Sigma_{g}^{+}$electron impact excitation by monokinetic electron beam experiments showed a negligibly small change of angular momentum $\Delta N \equiv N^{\prime}-N$ for $\nu^{\prime}=2$. Among the other diagonal $\left(\nu^{\prime}=\nu^{\prime \prime}\right)$ bands of the Fulcher- $\alpha$ system the $\left[\nu^{\prime}=\nu^{\prime \prime}=2\right]$ branch has been recommended as the most suitable for determination of the rotational and gas temperatures [21].

In order to prove that the observed electronic transition has a resolved rotational structure in our discharge, first the Fortrat parabolas of all branches versus the corresponding line wave numbers are constructed and drawn in Fig. 1.
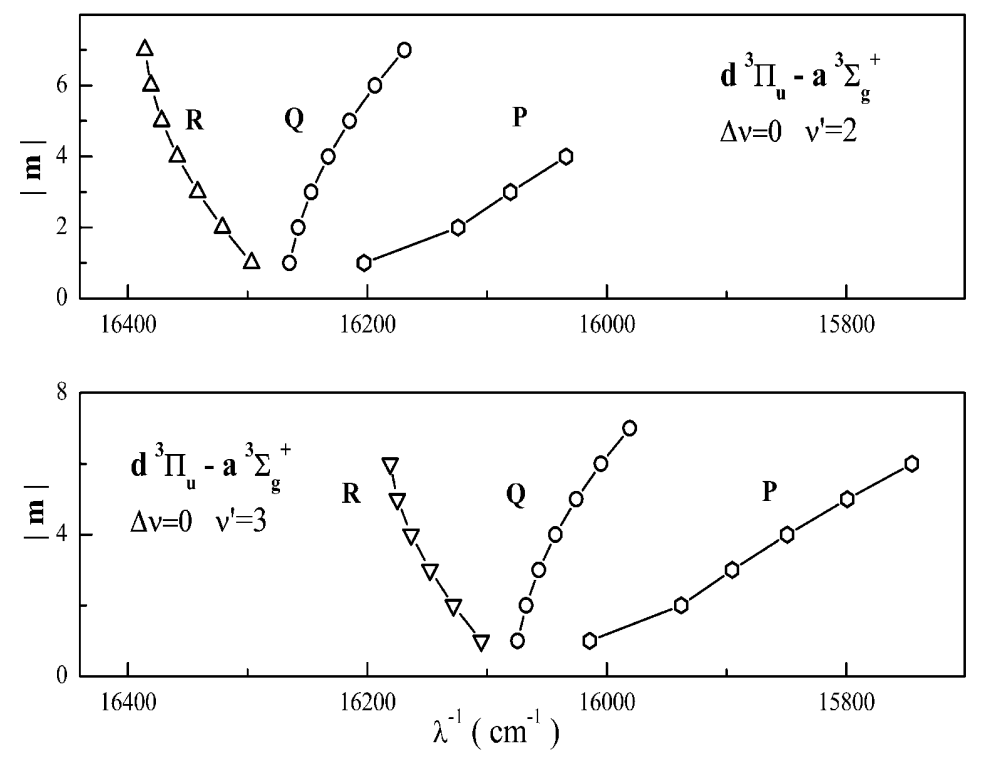

Figure 1. Fortrat parabolas of the deuterium Fucher- $\alpha$ bands for $\left[\nu^{\prime}=\nu^{\prime \prime}=2\right]$ and $\left[\nu^{\prime}=\nu^{\prime \prime}=3\right]$, where $m$ is an integer, which counts the successive lines in each branch. 
From the example of the recorded spectra in Fig. 2 and Fig. 3 it is evident that Q-branch lines of the $d^{3} \Pi_{u}^{-} \rightarrow a^{3} \Sigma_{g}^{+}\left[\nu^{\prime}=\nu "=2,3\right]$ electronic transition are well resolved and have high intensities in the $610-630 \mathrm{~nm}$ wavelength region (wavelength data are taken from [31]). For the $d^{3} \Pi_{u}$ state, the triplet splitting is negligible due to the small value of the spin-orbit interaction constant, which corresponds to a nearly pure Hund's case (b) coupling. The $a^{3} \Sigma_{g}^{+}$state is well described by a pure Hund's case (b) coupling and the rotational fine structure can be neglected $[29,32]$.

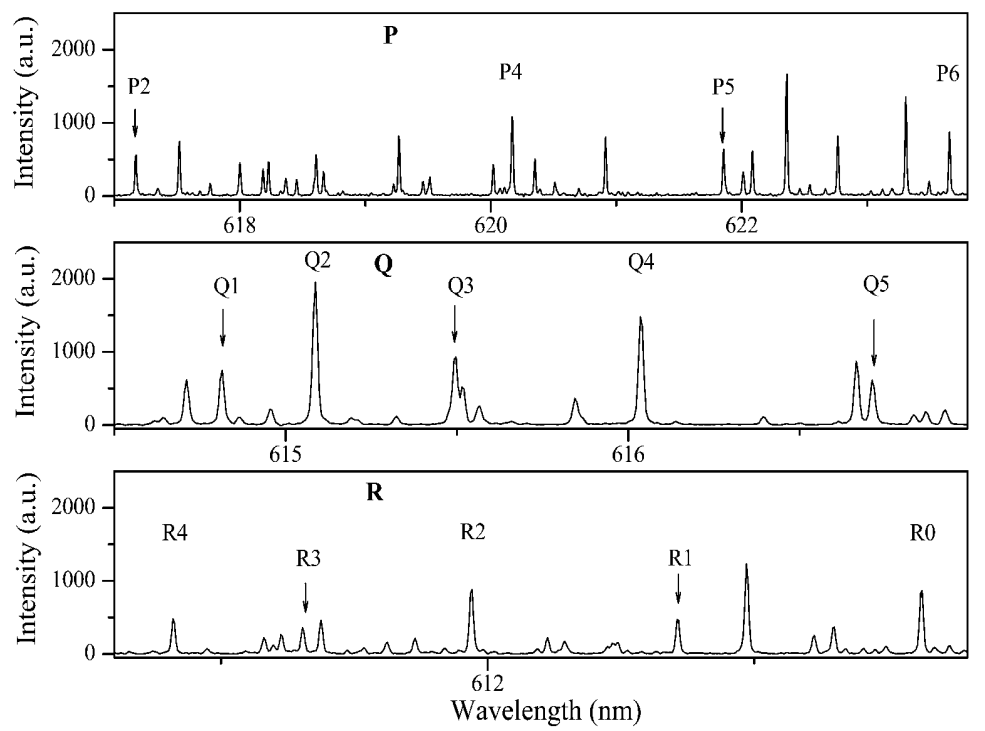

Figure 2. Emission spectra of rotational lines for $d^{3} \Pi_{u} \rightarrow a^{3} \Sigma_{g}^{+}$system; P-, Q- and R-branches with $\Delta \nu=0, \nu^{\prime}=2$. Experimental conditions: titanium $\mathrm{HC}$ discharge in $\mathrm{D}_{2}$ at $p=2.5$ mbar; $I=90 \mathrm{~mA} ; U=490 \mathrm{~V}$.

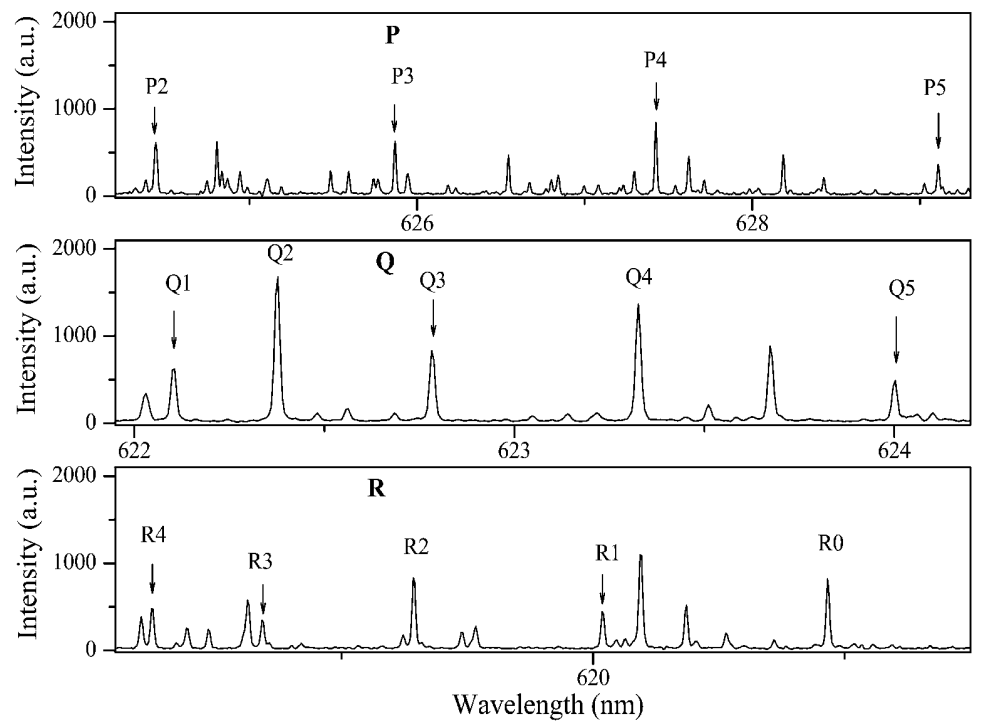

Figure 3. Emission spectra of rotational lines for $d^{3} \Pi_{u} \rightarrow a^{3} \Sigma_{g}^{+}$system; P-, Q- and R-branches with $\Delta \nu=0, \nu^{\prime}=3$. Experimental conditions: titanium $\mathrm{HC}$ discharge in $\mathrm{D}_{2}$ at $p=2.5 \mathrm{mbar}$; $I=90 \mathrm{~mA} ; U=490 \mathrm{~V}$. 
Although the spectral resolution and signal to noise ratio of our spectrometer-detector system enabled identification of $\mathrm{P}$ - or R-branch lines, their intensities are between one and two orders of magnitude weaker than those from the Q-branch of the same ro-vibrational transition, see the lowest part of Fig. 2.

In this study, the applicability of HL factors within $d^{3} \Pi_{u}^{+}, 2 \rightarrow a^{3} \Sigma_{g}^{+}, 2$ and $d^{3} \Pi_{u}^{+}, 3 \rightarrow$ $a^{3} \Sigma_{g}^{+}, 3$ transitions is investigated by means of the procedure described in [30]. Since the transition probabilities are unknown for the rotationally resolved ro-vibrational transitions, the HL factors will be used as line strengths. In such case, the so-called rotational branching ratio must be determined experimentally from line intensity ratios correlated with the relative line strengths $S_{R} / S_{P}$. The example reported in [31] illustrates well the described procedure. The ratios of measured line intensities within a pair of lines (in R- and P-branch) starting from the same rotational levels (see e.g. Grotrian diagram for Fulcher- $\alpha$ band transition, $\nu^{\prime}=\nu^{\prime \prime}=0$ ) in Fig. 2(a) of [20] has been used as a good indicator for applicability of HL factors for temperature measurements within $d^{3} \Pi_{u}^{+}, 2 \rightarrow a^{3} \Sigma_{g}^{+}, 2$ and $d^{3} \Pi_{u}^{+}, 3 \rightarrow a^{3} \Sigma_{g}^{+}, 3$ transitions.

The ratio values predicted by the HL formulae for Fulcher- $\alpha$ bands $\left[\Delta \nu=0, \nu^{\prime}=2\right]$ and $\left[\Delta \nu=0, \nu^{\prime}=3\right]$ are plotted in Fig. 4 as a function of the rotational quantum number. The comparison of data shows that the experimental ratio of line intensities for $\mathrm{R}$ and $\mathrm{P}$ follows the trend predicted by the HL formulae.
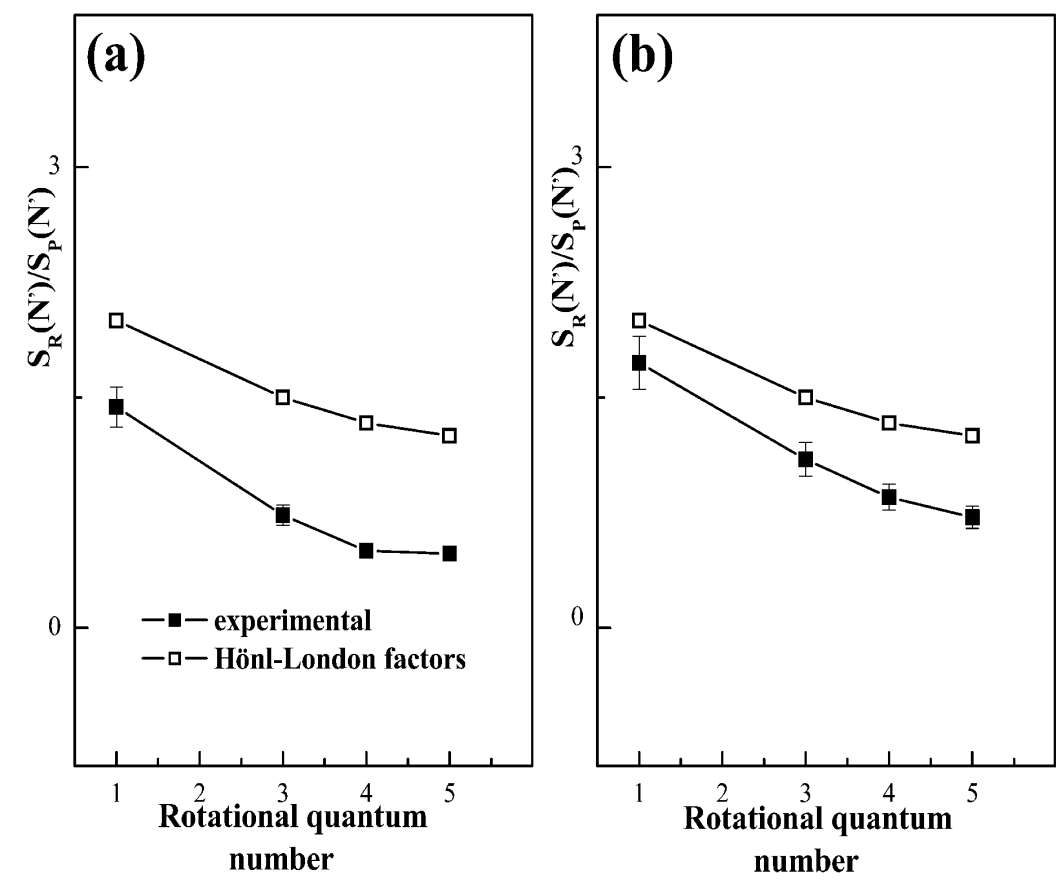

Figure 4. The comparison of measured branching ratios with the ratios of Hönl-London (HL) factors for: (a) $d^{3} \Pi_{u}^{+}, 2 \rightarrow a^{3} \Sigma_{g}^{+}, 2$ and (b) $d^{3} \Pi_{u}^{+}, 3 \rightarrow a^{3} \Sigma_{g}^{+}, 3$ transitions. Discharge conditions: as in Fig. 2.

The quantitative interpretation of the phenomenon when the experimental branching ratios are in large discordance with those calculated by HL formulae is given on the basis of nonadiabatic theory of the upper state rotational levels perturbation by other electronicvibration states of the molecule [29,33]. On the other hand, under our experimental 
conditions, another possible cause can be the fact that the intensities of $\mathrm{P}$ - and R-branch lines are one to two orders of magnitude lower than those of the Q-branch.
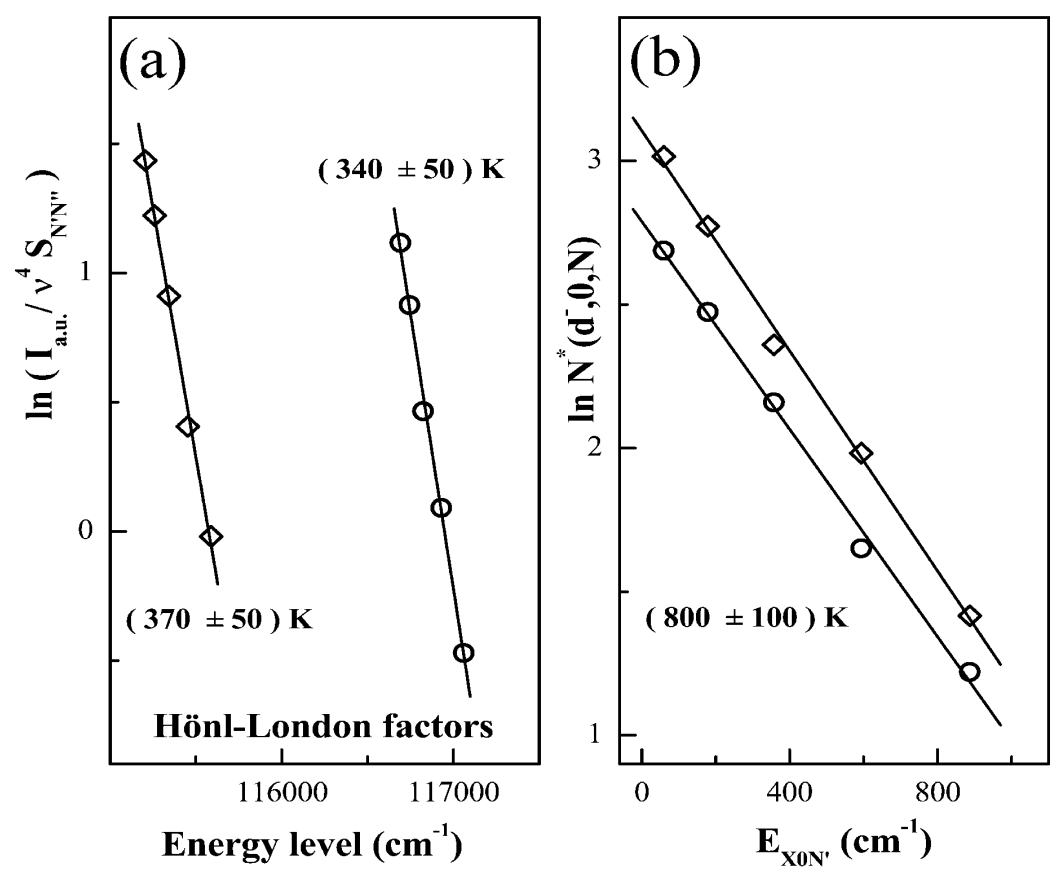

Figure 5. (a) A Boltzmann plot of line intensities calculated with Hönl-London factors for: $d^{3} \Pi_{u}^{-}, 2, N^{\prime}$ and $d^{3} \Pi_{u}^{-}, 3, N^{\prime}$ and (b) plots of rotational levels population densities versus rotational energy of the molecular deuterium ground state $\left(X^{1} \Sigma_{g}^{+}, 0\right)$ of: $d^{3} \Pi_{u}^{-}, 2, N^{\prime}$ and $d^{3} \Pi_{u}^{-}, 3, N^{\prime}$ rovibronic levels of $\mathrm{D}_{2}$; determined temperatures are indicated. Discharge conditions: as in Figs. 2 and 3 .

It is evident from Fig. 5 that the temperature obtained using limited number of spectral lines from the Q-branch of $\left[\nu^{\prime}=\nu^{\prime \prime}=2\right]$ Fulcher- $\alpha$ band agrees well with the temperature measured from the Q-branch of $\left[\nu^{\prime}=\nu^{\prime \prime}=3\right]$ Fulcher- $\alpha$ band. The rotational temperature is derived from the population of the Q-branch of $\mathrm{D}_{2}$ Fulcher- $\alpha$ bands $\left[\nu^{\prime}=\nu^{\prime \prime}=2\right]$ and $\left[\nu^{\prime}=\nu^{\prime \prime}=3\right]$ using HL factors and data from [31].

Thus, values of the rotational temperature derived from the Q-branch population in $d^{3} \Pi_{u}^{-}, 2 \rightarrow a^{3} \Sigma_{g}^{+}, 2$ and from $d^{3} \Pi_{u}^{-}, 3 \rightarrow a^{3} \Sigma_{g}^{+}, 3$ were used in conjunction with (1) to determine $T_{0}$ of the ground vibronic state $X^{1} \Sigma_{g}^{+},(\nu=0)$. The rotational energies $E_{X O N^{\prime}}$ of the ground $X^{1} \Sigma_{g}^{+},(\nu=0)$ vibronic state of $\mathrm{D}_{2}$ were listed in [31]. The results in Fig. 5 (b) show both temperatures which agree well within the estimated experimental uncertainties.

The values of the rotational temperature derived from the listed bands at different radial position, see Fig. 6. (b), show that the temperature changes along the HC diameter, as well as the radial intensity distribution of the deuterium Fulcher- $\alpha$ system with the maximum at $\mathrm{HC}$ axis.

The experimental lifetime values for ro-vibrational levels of electronic state $d^{3} \Pi_{u}^{-}[34]$ of the $\mathrm{D}_{2}$ molecule have been used to calculate radiative decay of this state which is $\approx 2.5 \times 10^{7} \mathrm{~s}^{-1}$. Under similar experimental conditions, but for hydrogen discharges [35], 
neglecting the collision quenching, the collision frequency is lower than the radiative decay of this state and the number of collisions is not sufficient to redistribute the rotational population. The pressure larger then 10 mbar would provide collision frequency larger than the radiative decay and the neutral species rotational collision mixing during the lifetime of the $d^{3} \Pi_{u}^{-}$state would be sufficient to ensure equilibrium of the rotational distribution of this state with the gas temperature.

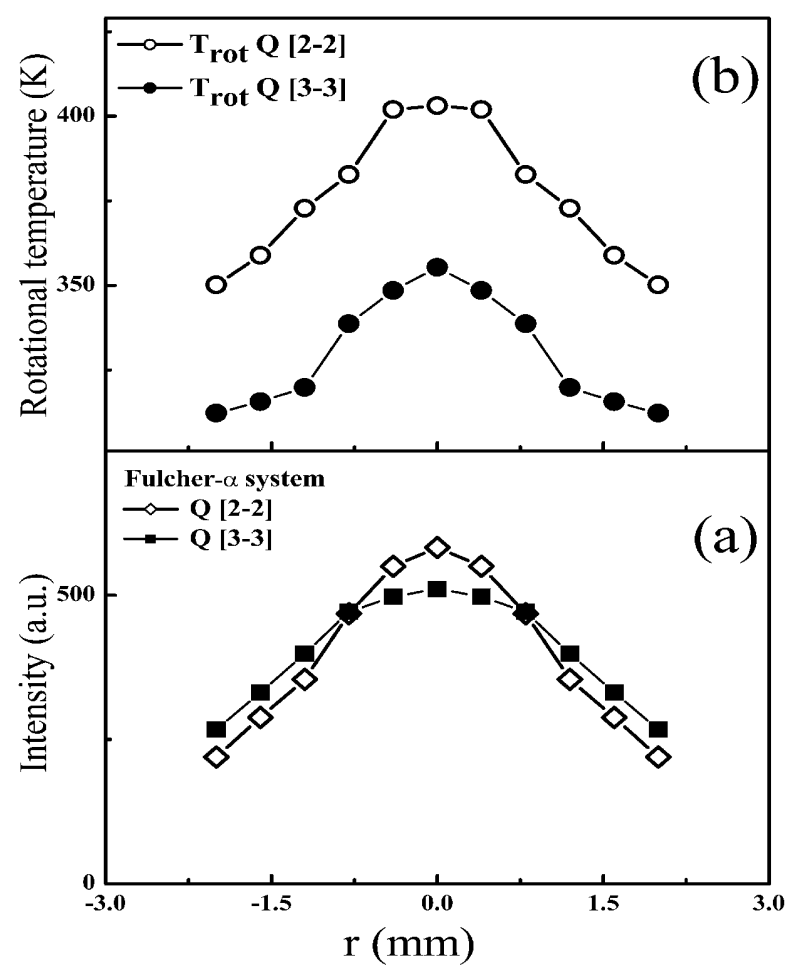

Figure 6. (a) Radial intensity distribution of the Fulcher- $\alpha$ system $\left(d^{3} \Pi_{u}^{-}, 2 \rightarrow a^{3} \Sigma_{g}^{+}, 2\right.$ electronic transition; Q-branch) (b) Calculated rotational $\mathrm{D}_{2}$ temperature distribution. Experimental conditions: titanium $\mathrm{HC}$ discharge in pure $\mathrm{D}_{2}$ at $p=2.5 \mathrm{mbar} ; I=90 \mathrm{~mA} ; U=490 \mathrm{~V}$.

In accordance with the above described model of temperature measurement in low pressure deuterium discharge, the gas temperature is expected to be twice as large as the rotational temperature of an excited state, see Eq. (1), where rotational constants of excited and ground vibronic states are correlated, see Eq. (2). In our case, the temperature recalculated from (1) for the ground vibronic state $X^{1} \Sigma_{g}^{+},(\nu=0)$ is two times larger than the rotational temperature of excited states $d^{3} \Pi_{u}, 2$ and $d^{3} \Pi_{u}, 3$.

The test of HL factors applicability for $d^{3} \Pi_{u}, 2 \rightarrow a^{3} \Sigma_{g}^{+}, 2$ and $d^{3} \Pi_{u}, 3 \rightarrow a^{3} \Sigma_{g}^{+}, 3$ transitions prove that in these cases non-adiabatic effects don't exist. Thus, to use the $d^{3} \Pi_{u}, 2 \rightarrow a^{3} \Sigma_{g}^{+}, 2$ band for determination of the gas temperature in gas discharges, it is not necessary to know the transition probabilities.

The result of measuring deuterium rotational temperature by means of a limited number of spectral lines belonging to two different bands at lower pressure, show an agreement between the rotational $T_{\text {rot }}(d, 2 Q)$ and $T_{\text {rot }}(d, 3 Q)$ temperatures as well as between $T_{0}(d, 0)$ of the ground vibronic state $X^{1} \Sigma_{g}^{+},(\nu=0)$. For the measurement of rotational 
and ground state $T_{0}$ temperature it is possible to use the first five lines of the Q-branch of $d^{3} \Pi_{u}, 2 \rightarrow a^{3} \Sigma_{g}^{+}, 2$ band.

\section{Summary}

The OES technique is employed to determine $\mathrm{D}_{2}$ molecule rotational $T_{\text {rot }}$ and translational temperature $T_{\mathrm{tr}}$ in HCGD, operated in deuterium with titanium cathode. The comparison between measured and HL factors branching ratios for Fulcher- $\alpha$ (2-2) and (3-3) band is performed.

The rotational temperature derived from the population of the Q-branch of Fulcher- $\alpha$ (2-2) and (3-3) band is approximately the same within estimated uncertainty (360 \pm 50$) \mathrm{K}$. In conjunction with (1) we determined $T_{0}$ of the ground vibronic state $X^{1} \Sigma_{g}^{+},(\nu=0)$, which is assumed to be equal to $T_{\mathrm{tr}}$. The molecular deuterium temperature $T_{0}\left(d^{-}, 0\right)$ of Ti HCGD is $(800 \pm 100) \mathrm{K}$. Under present experimental conditions the temperature $T_{0}$ for the ground vibronic state $X^{1} \Sigma_{g}^{+},(\nu=0)$ of the deuterium molecule is two times larger than the rotational temperatures of the excited states $d^{3} \Pi_{u}$.

\section{Acknowledgments}

This work is supported by the Ministry of Education and Science of the Republic of Serbia under Project No. 171014.

\section{References}

[1] P. J. Slevin and W. W. Harrison, Appl. Spectr. Rev. 10(2), 201 (1975).

[2] C. F. Da Silva, T. Ishikawa, S. Santos, Jr. C. Alves, A. E. Martinelli, International Journal of Hydrogen Energy. 31, 49 (2006).

[3] S. Krasheninnikov, Phys. Scr. T 96, 7 (2002).

[4] E. M. Hollmann, A. Yu. Pigarov and K. J. Taylor, Nucl. Mater. 337-339, 451 (2005).

[5] M. P'ealat, J-P. E. Taran, M. Bacal, F. Hillion, J. Chem. Phys. 82(11), 4943 (1985).

[6] V. A. Shakhatov, O. De Pascale, M. Capitelli, K. Hassouni, G. Lombardi, and A. Gicquel, Physics of Plasmas 12, 23504 (2005).

[7] H. N. Chu, E. A. Den Hartog, A. R. Lefkow, J. Jacobs, L. W. Anderson, M. G. Lagally and J. E. Lawler, Phys. Rev. A 443796 (1991).

[8] A. Gicquel, K Hassouni, Y. Breton, M. Chenevier and J. C. Cubertafon, Diam. and Rel. Mater. 5, 366 (1996).

[9] J. Laimer, F. Huber, G. Misslinger and H. Störi, Vacuum 47, 183 (1996).

[10] L. Tomasini, A. Rousseau, G. Gousset and P. J. Leprince, J. Phys. D: Appl Phys. 29, 1006 (1996). 
[11] S. A. Astashkevich, M. V. Kalachev, B. P. Lavrov, V. L. Ovtchinnikov, Opt. Spectr. 87, 203 (1999).

[12] J. Röpcke, P. B. Davies, M. Käning and B. P. Lavrov, Low temperature plasma physics-fundamental aspects and application ( Wiley-VCH Berlin, NY Toronto etc: Hippler R at al., 2001).

[13] B. Xiao, S. Kado, S. Kajita, D. Yamasaki and S. Tanaka, J. Nucl. Mater. 337-339, 1082 (2005).

[14] A. Pospieszczyk, S. Brezinsek, G. Sergienko et al., J. of Nucl. Mat. 337-339, 500 (2005).

[15] B. P. Lavrov, A. V. Pipa, and J. Röpcke, Plasma Sources Sci. Technol. 15, 135 (2006).

[16] U. Fantz, B. Heger, Plasma Phys. Control. Fusion 40, 2023 (1998).

[17] B. P. Lavrov, M. Osiac, A. V. Pipa, J. Röpcke, Plasma Source Sci. Technol. 12, 576 (2003).

[18] E. Surrey, B. Crowley, Plasma Phys. Control. Fusion 45, 1209 (2003).

[19] S. Kado, D. Yamasaki, B. Xiao, Y. IIda, A. Okamoto, S. Kajita, T. Shikama, T. Oishi, S. Tanaka, J. Plasma Fusion Res. SERIES 7, 54 (2006).

[20] G. Lj. Majstorović, N. M. Šišović and N. Konjević, Plasma Sources Sci. Technol. 16, 750 (2007).

[21] M. Osiac, B. P. Lavrov and J. Röpcke, J. Q. S. R. T. 74, 471 (2002).

[22] N. M. Šišović, G. Lj. Majstorović and N. Konjević, Eur. Phys. J. D 32, 347 (2005).

[23] M. Abdel-Rahman, T. Gans, V. Schulz-von der Gathen and H. F. Döbele, Plasma Sources Sci. Technol. 14, 51 (2005).

[24] S. A. Astashkevich, M. Käning, E. Käning, N. V. Kokina, B. P. Lavrov, A. Ohl and J. Röpcke, J. Q. S. R. T. 56, 725 (1996).

[25] G. Herzberg G., Molecular Spectra and Molecular Structure, Spectra of Diatomic Molecules Vol. 1 (Krieger Publishing Co, Malabar FL 1989).

[26] I. Covacs, Rotational structure in the spectra of diatomic molecules, p. 122, (London: Adam Hilger LTD, 1969).

[27] B. P. Lavrov, Opt. Spectrosc. 48, 375 (1980).

[28] A. I. Drachev and B. P. Lavrov, High. Temp. 26, 129 (1988).

[29] D. R. Farley, D. P. Stotler, D. P. Lundberg, S. A. Cohen J. Q. S. R. T. 112, 800-819 (2011).

[30] B. P. Lavrov, Plasma Chemistry, p. 45, (Smirnov BM ed. Moscow: Energoatomizdat, 1984). 
[31] H. M. Crosswhite, R. S. Freund, J. A. Schiavone and H.M. Crosswhite J. Chem.Phys.Ref. Data 14, 235 (1985).

[32] R. Wijtvliet, E. Felizardo, E. Tatarova, F. M. Dias, C. M. Ferreira, S. Nijdam, E. V. Veldhuizen and G. Kroesen, Journal of Applied Physics 106, 103301 (2009).

[33] D. Gleispach, A. Winkler, Surface Science 537, L435L441 (2003)..

[34] arxiv.org/pdf/1412.3396

[35] C. Barbeau, G. Baravian and J. Jolly, X Int. Symp. on Plasma Chemistry (Bochum) (1991). 